\title{
Métodos de interpolações de dados obtidos por sensores de agricultura de precisão
}

\author{
Emanuele Helmann NUNES ${ }^{1 *}$, Thiago Martins MACHADO ${ }^{1}$, Étore Francisco REYNALDO ${ }^{2}$, \\ Cassiano Spaziani PEREIRA ${ }^{1}$
}

\author{
${ }^{1}$ Programa de Pós-Graduação em Agronomia, Instituto de Ciências Agrárias e Ambientais, \\ Universidade Federal de Mato Grosso, Sinop, MT, Brasil. \\ ${ }^{2}$ Bayer, Uberlândia, MG, Brasil. \\ *E-mail: manu.helmann@hotmail.com
}

Recebido em agosto/2017; Aceito em agosto/2018.

RESUMO: Os sensores que medem as características do solo em campo são importantes ferramentas para o manejo da agricultura de precisão, entre eles, destaca-se o sensor de contato, que mede a condutividade elétrica (CE), matéria orgânica (MO) e potencial hidrogeniônico $(\mathrm{pH})$ do solo. Objetivou-se avaliar os erros dos métodos de interpolação por krigagem, inverso da distância e normal da distância a partir de dados de CE, MO e pH do solo. O experimento foi realizado no município de Candói - PR, onde foram amostradas duas áreas, os dados foram coletados por sensor de contato, o qual foi conFigurado para uma coleta de 150 pontos por hectare para a condutividade elétrica e a matéria orgânica, e para potencial hidrogeniônico a frequência de coleta foi de 15 pontos por hectare, o equipamento foi acoplado em um trator operado a uma velocidade de $8 \mathrm{~km} \mathrm{~h}^{-1} \mathrm{com}$ passadas paralelas de $20 \mathrm{~m}$. Realizaram-se análises variográficas, validação cruzada e elaboração de mapas. Os menores erros de interpolação ou "jack knifing" para $\mathrm{CE}, \mathrm{MO}$ e $\mathrm{pH}$ foram apresentados pelo método de interpolação inverso da distância, para o talhão T2, e no talhão T1 o método da Krigagem obteve os menores erros para o pH. Concluiu-se que distância das amostragens foi adequada e a krigagem e o inverso da distância foram mais eficientes que o normal da distância. Verificou-se que quanto maior a potência de elevação, tanto para o método do inverso da distância quanto para normal da distância, os erros aumentam e também o grau de contagiosidade.

Palavras-chave: geoestatística, sensor Veris, variabilidade espacial.

\section{Methods of data interpolations obtained by precision agriculture sensors}

\begin{abstract}
The sensors that measure soil characteristics in the field are important tools for the management of precision agriculture, among them the contact sensor, which measures the electrical conductivity (EC), organic matter $(\mathrm{OM})$ and hydrogenation potential $(\mathrm{pH})$ of the soil. The objective of this study was to evaluate the errors of the interpolation methods by kriging, inverse distance and normal distance from the data of EC, $\mathrm{MO}$ and soil $\mathrm{pH}$. The experiment was carried out in the city of Candói - PR, where two areas were sampled, the data were collected by contact sensor, which was configured for a collection of 150 points per hectare for electrical conductivity and organic matter, and for potential the collection frequency was 15 points per hectare, the equipment was coupled in a tractor operated at a speed of $8 \mathrm{~km} \mathrm{~h}-1$ with parallel passes of $20 \mathrm{~m}$. Variographic analysis, cross-validation and mapping were performed. The smallest interpolation errors or jack knifing for $\mathrm{CE}, \mathrm{MO}$ and $\mathrm{pH}$ were presented by the inverse distance interpolation method for the T2 field, and in $\mathrm{T} 1$ field the Kriging method obtained the lowest errors for $\mathrm{pH}$. It was concluded that distance from the samplings was adequate and the kriging and the inverse of the distance were more efficient than the normal distance. It was verified that the higher the elevation power, both for the inverse distance and the normal distance method, the errors increase and also the degree of contagiousness.
\end{abstract}

Keywords: geostatistics, Veris sensor, spatial variability.

\section{INTRODUÇÃO}

Com os avanços da tecnologia nota-se a necessidade do aumento da produtividade das lavouras respeitando o meio ambiente, e para que isso ocorra é necessário aplicar os conceitos da agricultura de precisão. Segundo Manzatto et al. (1999), a agricultura de precisão consiste em aplicar os insumos no local correto, no momento adequado, e em quantidades necessárias.

Uma das dificuldades encontradas no campo é referente à quantidade de coletas de solo, geralmente a amostragem satisfatória geoestatisticamente é inviável pelo custo em laboratórios. De acordo com Carneiro et al. (2016) o alcance indica a distância máxima que a variável está correlacionada.
Vale ressaltar que os talhões a serem empregados a agricultura de precisão geralmente apresentam uma área muito grande, o que dificulta a coleta, necessitando de muito tempo para a realização da mesma em toda a área. De acordo com Cherubin (2014) a dependência espacial dos atributos do solo é menor do que a imaginada, ou seja, para obter a caracterização eficiente da distribuição espacial dos atributos do solo, em sua maioria, são necessárias amostragens densas.

De acordo com Bernardi et al. (2015) na densidade amostral existem grandes diferenças entre a coleta tradicional do solo e o uso de sensores, sendo que com a utilização dos sensores é possível cobrir de forma muito mais detalhada a área de estudo. 
A realização de amostragens em campo está sendo cada vez mais estudada. As técnicas mais utilizadas estão relacionadas à condutividade elétrica - CE (CORASSA et al., 2016), potencial hidrogeniônico - pH (MOLIN et al., 2015) e matéria orgânica - MO (KODAIRA; SHIBUSAWA, 2013).

Uma das ferramentas da agricultura de precisão é o uso do sensor Veris, sensor de contato que mede a condutividade elétrica e o potencial hidrogeniônico, e estima a matéria orgânica do solo, a sua utilização contribui para a agilidade de obtenção de informações em campo e em tempo real, e apresenta vantagens econômicas para quem o utiliza.

Os dados coletados em campo pelo sensor Veris são utilizados para confecção de mapas, os quais usam métodos de interpolação para estimar os valores não-amostrados, sendo que esses métodos apresentam erros, podendo um método apresentar um erro maior que outro. Segundo Bernardi et al. (2015) a partir dos modelos ajustados pelos semivariogramas, interpolam-se os dados gerando os mapas, as interpolações podem ser realizadas por vários métodos, os quais geram mapas de probabilidade, que são compostos por valores preditos e erros, de acordo com Cunha et al. (2013) esses mapas referem-se ao desvio padrão da predição para um ponto individual.

$\mathrm{Na}$ área de agricultura de precisão, tem se pouca informação sobre os melhores métodos e modelos de interpolação de dados para atributos do solo, que em muitas das vezes ocasiona uma confusão por parte do usuário de programas, de qual método e modelo a escolher. Sendo assim, objetivo deste estudo foi o de avaliar os erros, modelos e o melhor método de interpolação entre krigagem, inverso da distância e normal da distância, analisando os dados de condutividade elétrica, matéria orgânica e potencial hidrogeniônico do solo para mapeamento da variabilidade espacial, utilizando os dados coletados pelo sensor Veris.

\section{MATERIAL E MÉTODOS}

O experimento foi realizado no município de Candói, PR na fazenda Caracu, Grupo Santa Clara, em dois talhões, cujas coordenadas são, T1 (25³4'14” S, 52 $03^{\prime} 10^{\prime}$ W), T2 (2556'51" S, 51 $\left.28^{\prime} 47^{\prime \prime} \mathrm{W}\right)$.

A altitude média no local era $930 \mathrm{~m}$, o clima subtropical úmido mesotérmico segundo a classificação climática de Koppen-Geiger, com temperaturas médias de $28^{\circ} \mathrm{C}$ para máximas e menores que $14^{\circ} \mathrm{C}$ para mínimas. A lâmina média anual é de $1801,5 \mathrm{~mm}$, com chuvas bem distribuídas o ano todo. Os solos das áreas experimentais são classificados segundo (EMBRAPA, 2012) como Latossolo Bruno alumínico câmbico, com horizonte A proeminente, com classe textural variando de argilosa a muito argilosa. As áreas vêm sendo manejadas sob sistema plantio direto a mais de 15 anos, e cultivada com trigo, aveia ou cevada no inverno e soja ou milho no verão, em esquema de rotação de culturas.

Os dados foram coletados em dois talhões, T1 com 148,4 ha, e T2 com 111,6 ha. O teor de água no talhão T1 era de $27 \%$ e o teor de argila de $65,3 \%$, no talhão T2 o teor de água era de $29 \%$ e o teor de argila $60 \%$. O sensor utilizado foi o Sensor Veris PMC, sensor de contato que mede a condutividade elétrica, matéria orgânica e potencial hidrogeniônico do solo. Foi conFigurado para uma coleta de 150 pontos por hectare para a condutividade elétrica e a matéria orgânica, e para potencial hidrogeniônico a frequência de coleta foi de 15 pontos por hectare. Os dados coletados foram filtrados e apresentaram frequência de coleta de 122,120 e 14 pontos por hectare para o talhão T1, e 76, 75 e 9 pontos por hectare para o talhão T2, para a condutividade elétrica, matéria orgânica e potencial hidrogeniônico, respectivamente. $\mathrm{O}$ equipamento foi acoplado em um trator John Deere $7195 \mathrm{~J}$, operado a uma velocidade de $8 \mathrm{~km} \mathrm{~h}^{-1}$ com passadas paralelas de $20 \mathrm{~m}$.

A medição da condutividade elétrica consiste na emissão de uma corrente elétrica por dois discos intermediários, enquanto os dois discos internos e dois discos externos detectam a diferença de potencial, resultante da corrente elétrica aplicada, o par de discos internos faz a medição nas profundidades de 0 e $30 \mathrm{~cm}$, e o par de discos externos entre 0 e $90 \mathrm{~cm}$ (MACHADO et al., 2016).

O equipamento é capaz de quantificar o material orgânico, seu princípio de funcionamento consiste no uso da refletância para medir o teor de matéria orgânica no solo, que conta com dois espectrômetros integrados que realizam leituras simultâneas nas faixas de 350-2.200 nm com resolução espectral de $8 \mathrm{~nm}$ (CHIG, 2015). O equipamento opera em campo e em movimento, enquanto uma haste rompe o solo, outra haste realiza a leitura de refletância no fundo do sulco.

As leituras de potencial hidrogeniônico são realizadas por um sensor eletroquímico, que consiste em um amostrador de solo que coleta amostras, e cada amostra entra separadamente em contato direto com dois eletrodos de potencial hidrogeniônico. A amostra é dispensada e os eletrodos recebem um jato de água para limpeza, reiniciando o processo (MOLIN et al., 2015).

Inicialmente, realizaram-se as análises estatísticas descritivas dos dados, para análise do comportamento dos dados, calculou-se a média, mediana, desvio padrão, variância, coeficiente de variação, assimetria e curtose.

Para a realização das análises semivariográficas, validação cruzada e elaboração dos mapas foi utilizado o programa GS+7.0 (Geostatistics for the Environmental Sciences, Version 7). Sendo os modelos analisados: exponencial, Gaussiano, esférico e linear. O modelo escolhido foi o que resultou em um maior coeficiente de determinação, sendo ele, o modelo exponencial.

Quando a validação cruzada é realizada, a mesma gera valores estimados para a variável que está sendo analisada. Utilizaram-se esses dados estimados para comparar com os dados observados em campo, e assim calculou-se o erro absoluto médio (MAE - mean absolute error) dos modelos de interpolação, conforme a equação 1, descrita por WILLMOTT et al. (1985).

$$
M A E=\frac{\sum_{i=1}^{n}|P i-O i|}{n}
$$

em que: Pi: valores estimados; Oi: valores observados; n: número de dados amostrados.

A raiz quadrada da média do erro ao quadrado indica a acurácia, pois não utiliza a média, resultando no erro absoluto em relação à referência, representando $68 \%$ de uma distribuição. Calculou-se a raiz quadrada da média do erro (RMS - root mean square) conforme a equação 2, descrita por LEGATES; MCCABE (1999). 


$$
R M S=\sqrt{\frac{\sum_{i=1}^{n}(|P i-O i|)^{2}}{n}}
$$

\section{RESULTADOS}

A variabilidade dos atributos do solo foi avaliada pela análise exploratória dos dados. Na Tabela 1 são apresentadas as análises estatísticas descritivas dos valores de condutividade elétrica, matéria orgânica e potencial hidrogeniônico.

Tabela 1. Estatística descritiva para os valores de CE $\left(\mathrm{mS} \cdot \mathrm{m}^{-1}\right), \mathrm{pH}$ e MO $\left(\mathrm{g} \mathrm{kg}^{-1}\right)$ para as duas áreas experimentais.

Table 1. Descriptive statistics for the values of EC $\left(\mathrm{mS}^{-1} \mathrm{~m}^{-1}\right), \mathrm{pH}$ and $\mathrm{MO}\left(\mathrm{g} \mathrm{kg}^{-1}\right)$ for the two experimental areas.

\begin{tabular}{lllllll}
\hline & & T1 & & & T2 & \\
& CE & MO & pH & CE & MO & pH \\
\hline $\mathrm{N}^{\circ}$ amos & 13647 & 13406 & 1583 & 11262 & 11173 & 1273 \\
Mínimo & 1 & 1,93 & 5,05 & 0,10 & 1,89 & 4,95 \\
Máximo & 21,2 & 2,53 & 7,0 & 11,80 & 2,75 & 7,30 \\
Média & 4,34 & 2,12 & 6,18 & 3,72 & 2,16 & 6,07 \\
Mediana & 4 & 2,10 & 6,20 & 3,50 & 2,15 & 6,05 \\
DP & 1,60 & 0,10 & 0,41 & 1,05 & 0,09 & 0,45 \\
Variância & 2,53 & 0,01 & 0,17 & 1,10 & 0,01 & 0,20 \\
CV (\%) & 36,88 & 4,69 & 6,63 & 28,23 & 4,17 & 7,41 \\
Assimetria & 1,36 & 1,10 & $-0,2$ & 1,30 & 1,23 & 0,10 \\
Curtose & 3,53 & 1,02 & $-0,6$ & 4,44 & 3,49 & $-0,7$
\end{tabular}

Sendo: $\mathrm{N}^{\circ}$ amos: número de amostras. DP: desvio padrão. $\mathrm{CV}$ : coeficiente de variação.

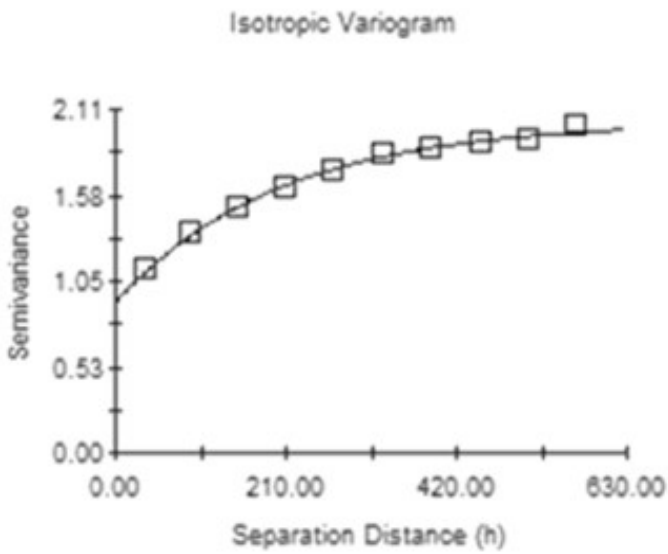

(a)

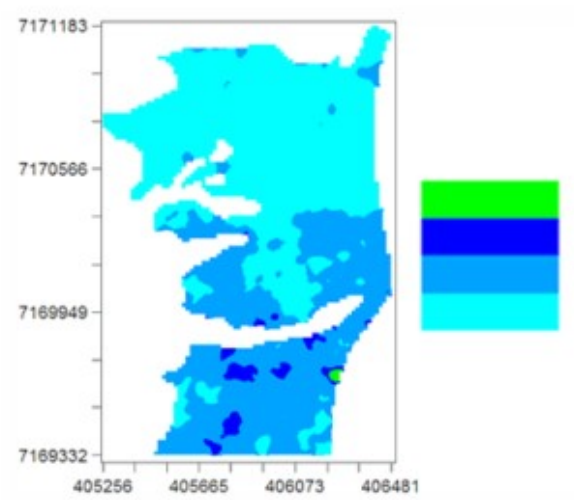

(b)

Figura 1. Modelo do semivariograma ajustado (a) e mapa para a condutividade elétrica do talhão T1 (b).

Figure 1. Model of the adjusted semivariogram (a) and map for the electrical conductivity of field T1 (b)
Das Figuras 1 a 6 são apresentadas o semivariograma, o mapa e a validação cruzada para a condutividade elétrica, matéria orgânica e potencial hidrogeniônico do talhão T1.

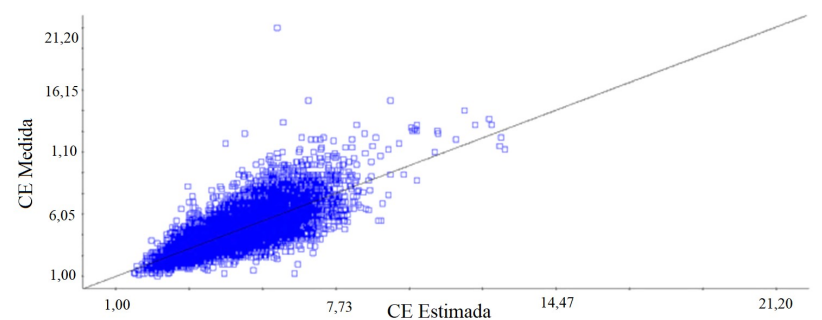

Figura 2. Validação cruzada entre condutividade elétrica medida e estimada para o talhão T1.

Figure 2. Cross-validation between measured and estimated electrical conductivity for field $\mathrm{T} 1$.

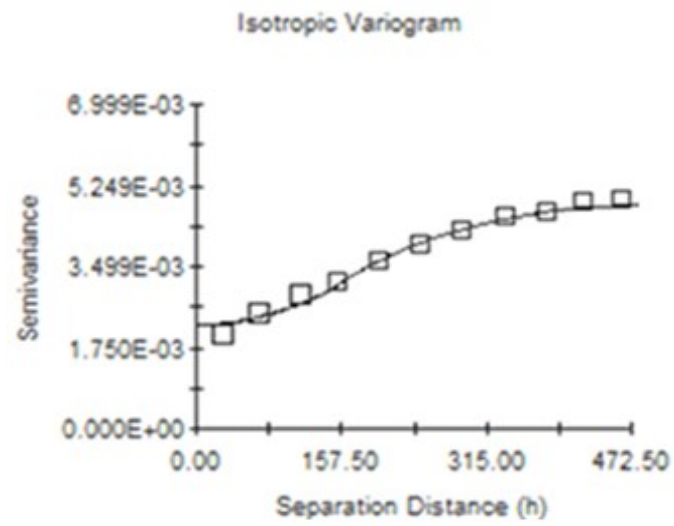

(a)

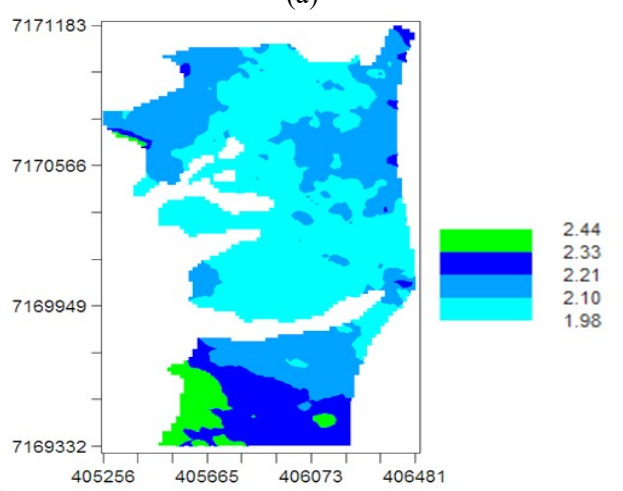

(b)

Figura 3. Modelo do semivariograma ajustado (a) e mapa para a matéria orgânica do talhão T1 (b).

Figure 3. Model of the adjusted semivariogram (a) and map for the organic matter of the plot $\mathrm{T} 1$ (b).

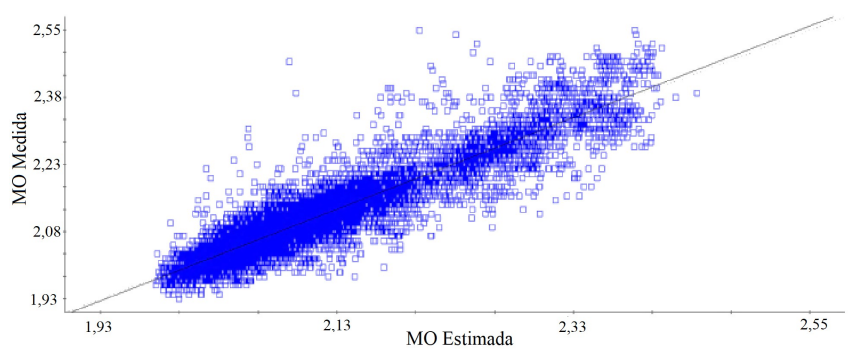

Figura 4. Validação cruzada entre matéria orgânica medida e estimada do talhão T1.

Figure 4. Cross-validation between measured and estimated organic matter of the T1 plot. 
Isotropic Variogram

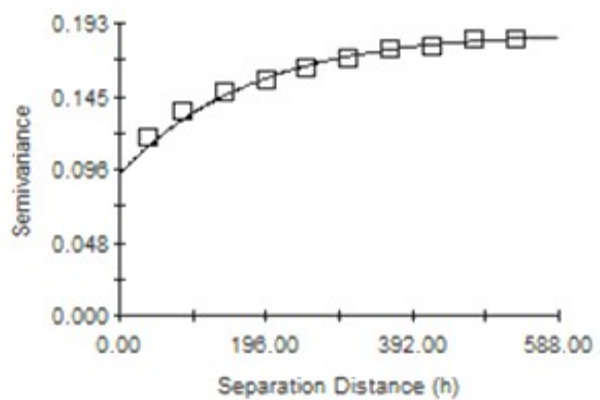

(a)

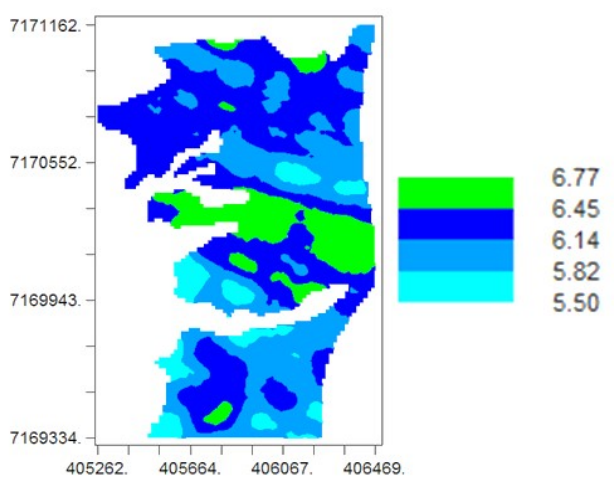

(b)

Figura 5. Modelo do semivariograma ajustado (a) e mapa para o potencial hidrogeniônico do talhão T1 (b).

Figure 5. Model of the adjusted semivariogram (a) and map for the hydrogenation potential of the T1 (b) plot.

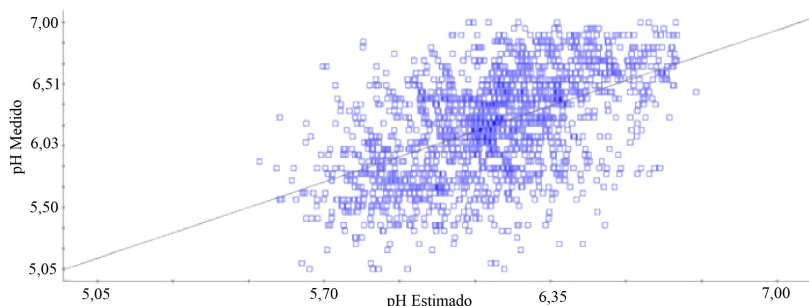

Figura 6. Validação cruzada entre potencial hidrogeniônico medido e estimado do talhão T1.

Figure 6. Cross-validation between measured and estimated hydrogenation potential of the T1 plot.

Das Figuras 7 a 12 são apresentadas o semivariograma, o mapa e a validação cruzada para a condutividade elétrica, matéria orgânica e potencial hidrogeniônico do talhão T2.

Nas Tabelas 2 e 3 são apresentados os parâmetros dos semivariogramas e da validação cruzada referentes aos valores de condutividade elétrica, matéria orgânica e potencial hidrogeniônico para o talhão T1, e nas Tabelas 4 e 5 são apresentados os parâmetros para o talhão T2.

Os atributos físicos químicos do solo apresentaram bons índices de coeficiente de determinação para o semivariograma, os modelos representam mais de $90 \%$ dos dados (Tabela 4).

Na Tabela 6 são apresentados os erros médios absolutos de interpolações dos métodos de krigagem, inverso da distância e normal da distância, para as variáveis de condutividade elétrica, matéria orgânica e potencial hidrogeniônico para os talhões T1 e T2.

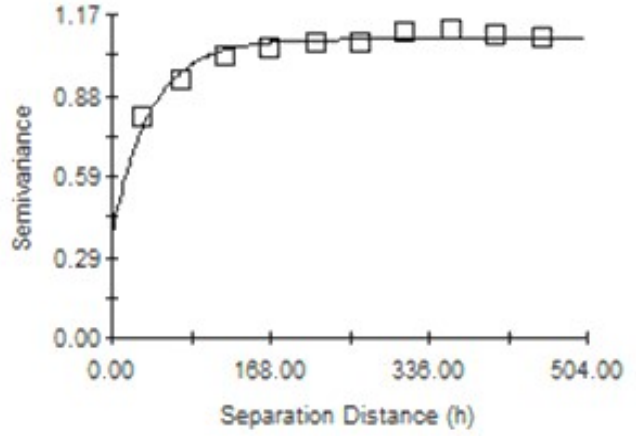

(a)

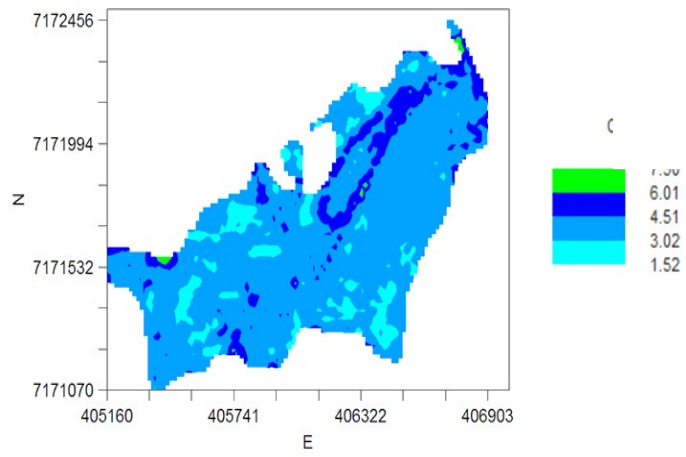

(b)

Figura 7. Modelo do semivariograma ajustado (a) e mapa para a condutividade elétrica do talhão T2 (b).

Figure 7. Model of the adjusted semivariogram (a) and map for the electrical conductivity of field T2 (b).

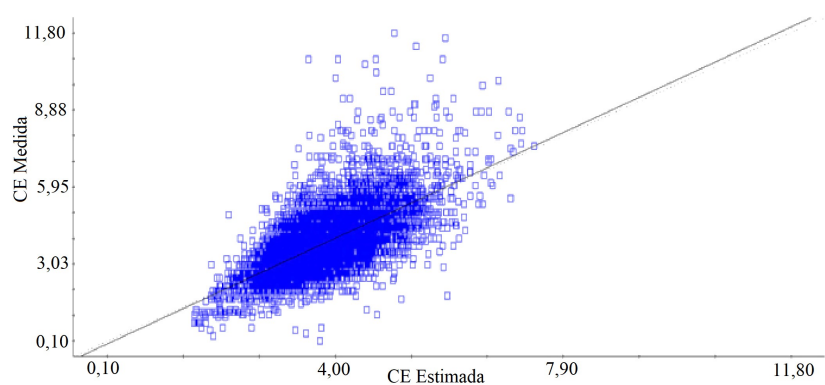

Figura 8. Validação cruzada entre a condutividade elétrica medida e estimada do talhão T2.

Figure 8. Cross-validation between the measured and estimated electrical conductivity of the $\mathrm{T} 2$ field.

Isotropic Variogram

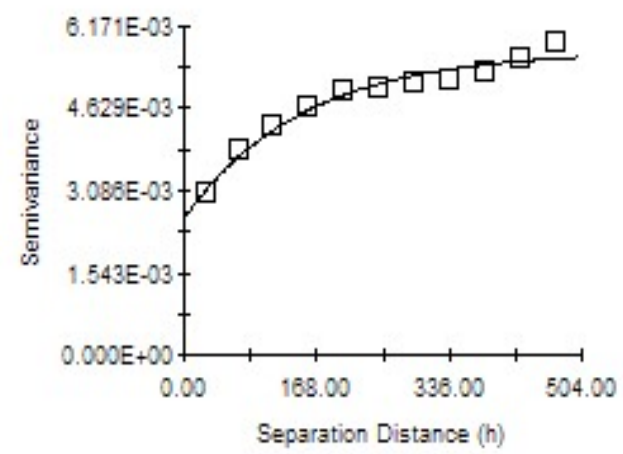

(a) 


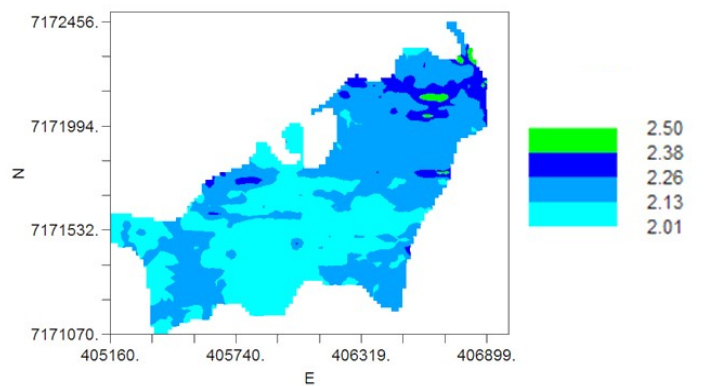

(b)

Figura 9. Modelo do semivariograma ajustado (a) e mapa para a matéria orgânica do talhão T2 (b).

Figure 9. Model of the adjusted semivariogram (a) and map for the organic matter of field T2 (b).

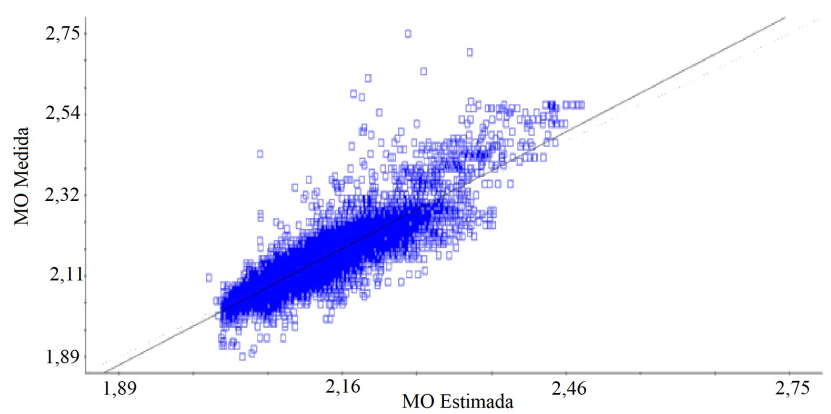

Figura 10. Validação cruzada entre a matéria orgânica medida e estimada do talhão T2.

Figure 10. Cross-validation between the measured and estimated organic matter of the $\mathrm{T} 2$ field.

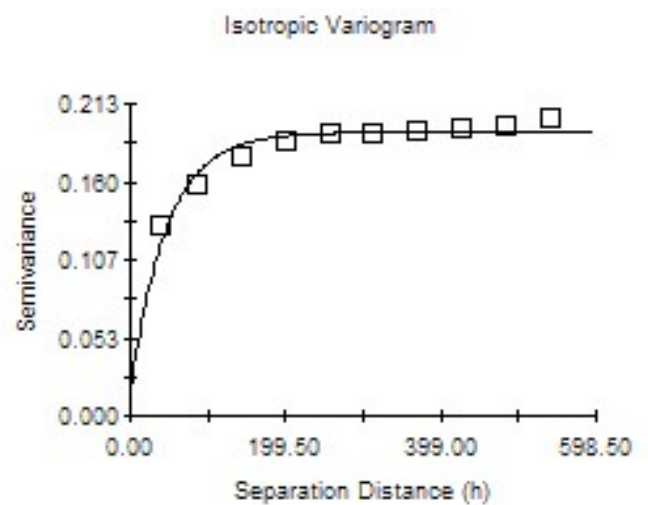

(a)

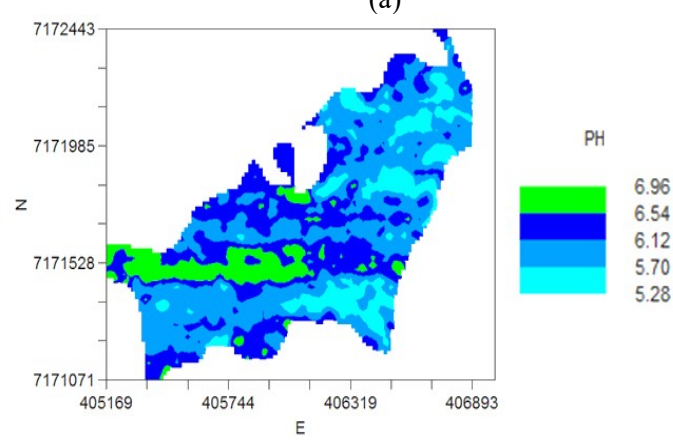

(b)

Figura 11. Modelo do semivariograma ajustado (a) e mapa para o potencial hidrogeniônico do talhão T2 (b).

Figure 11. Model of the adjusted semivariogram (a) and map for the hydrogenation potential of the T2 (b) plot.

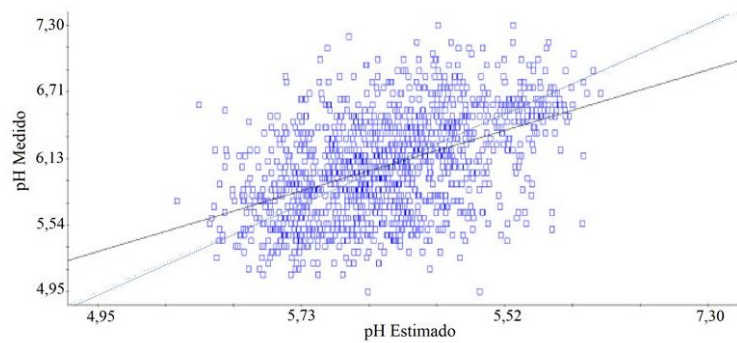

Figura 12. Validação cruzada entre o potencial hidrogeniônico medido e estimado do talhão $\mathrm{T} 2$.

Figure 12. Cross-validation between the measured and estimated hydrogenation potential of the $\mathrm{T} 2$ field.

Tabela 2. Parâmetros dos semivariogramas dos valores de $\mathrm{CE}$ $\left(\mathrm{mS} . \mathrm{m}^{-1}\right), \mathrm{pH}$ e $\mathrm{MO}\left(\mathrm{g}^{\mathrm{kg}} \mathrm{kg}^{-1}\right)$ para o talhão T1 utilizando sensor Veris PMC.

Table 2. Parameters of semivariograms of the values of CE (mS.m $\left.{ }^{1}\right), \mathrm{pH}$ and $\mathrm{MO}\left({\mathrm{g} . \mathrm{kg}^{-1}}^{-1}\right)$ for the T1 field using Veris PMC sensor.

\begin{tabular}{cccccccc}
\hline Var & Mod & $\mathrm{r}^{2}$ & Co & Co+C & A & GDE & R \\
\hline CE & Exp & 0,99 & 0,923 & 2,0330 & 256 & 0,546 & 0,00397 \\
MO & Exp & 0,95 & 0,002 & 0,0105 & 289 & 0,850 & 0,00001 \\
pH & Exp & 0,91 & 0,001 & 0,2041 & 232 & 0,995 & 0,0319 \\
\hline
\end{tabular}

Sendo: Var: variável. Mod: modelo. $\mathrm{r}^{2}$ : coeficiente de determinação. Co: efeito pepita. $\mathrm{Co}+\mathrm{C}$ : patamar. A: alcance. GDE: grau de dependência espacial. R: resíduo.

Tabela 3. Parâmetros da validação cruzada dos valores de $\mathrm{CE}$ $\left(\mathrm{mS} . \mathrm{m}^{-1}\right), \mathrm{pH}$ e $\mathrm{MO}\left(\mathrm{g} \cdot \mathrm{kg}^{-1}\right)$ para o talhão T1 utilizando sensor Veris PMC.

Table 3. Parameters of the cross-validation of the values of EC $\left(\mathrm{mS} . \mathrm{m}^{-1}\right), \mathrm{pH}$ and $\mathrm{MO}\left(\mathrm{g} . \mathrm{kg}^{-1}\right)$ for the T1 field using Veris PMC sensor.

\begin{tabular}{lcccc}
\hline Var & $\mathrm{r}^{2}$ & $\mathrm{CR}$ & $\mathrm{Y}$ & Erro \\
\hline $\mathrm{CE}$ & 0,632 & 1,004 & $-0,01$ & 0,966 \\
$\mathrm{MO}$ & 0,853 & 1,023 & $-0,05$ & 0,038 \\
$\mathrm{pH}$ & 0,274 & 0,926 & 0,46 & 0,353 \\
\hline
\end{tabular}

Sendo: Var: variável, $\mathrm{r}^{2}$ : coeficiente de determinação, CR: coeficiente de regressão, Y: intercepto no eixo y, Erro: erro padrão previsto.

Tabela 4. Parâmetros dos semivariogramas dos valores de CE $\left(\mathrm{mS} . \mathrm{m}^{-1}\right), \mathrm{pH}$ e $\mathrm{MO}\left(\mathrm{g} \cdot \mathrm{kg}^{-1}\right)$ para o talhão $\mathrm{T} 2$ utilizando sensor Veris PMC.

Table 4. Parameters of the semivariograms of the values of EC $\left(\mathrm{mS} . \mathrm{m}^{-1}\right), \mathrm{pH}$ and $\mathrm{MO}\left(\mathrm{g} . \mathrm{kg}^{-1}\right)$ for the T2 field using Veris PMC sensor.

\begin{tabular}{cccccccc}
\hline Var & Mod & $\mathrm{r}^{2}$ & Co & Co+C & A & GDE & $\mathrm{R}$ \\
\hline CE & Exp & 0,93 & 0,373 & 1,0850 & 123 & 0,656 & 0,00616 \\
MO & Exp & 0,96 & 0,002 & 0,0057 & 183 & 0,548 & 0,00002 \\
pH & Exp & 0,92 & 0,016 & 0,1936 & 141 & 0,913 & 0,00042
\end{tabular}

Sendo: Var: variável. Mod: modelo, $\mathrm{r}^{2}$ : coeficiente de determinação, Co: efeito pepita. Co+C: patamar, A: alcance. GDE: grau de dependência espacial. R: resíduo.

Tabela 5. Parâmetros da validação cruzada dos valores de $\mathrm{CE}$ $\left(\mathrm{mS} . \mathrm{m}^{-1}\right), \mathrm{pH}$ e $\mathrm{MO}\left(\mathrm{g}^{\mathrm{kg}} \mathrm{kg}^{-1}\right)$ para o talhão T2 utilizando sensor Veris PMC.

Table 5. Parameters of the cross-validation of the values of EC $\left(\mathrm{mS} . \mathrm{m}^{-1}\right), \mathrm{pH}$ and $\mathrm{MO}\left(\mathrm{g} . \mathrm{kg}^{-1}\right)$ for the T2 field using Veris PMC sensor.

\begin{tabular}{ccccc} 
Var & $\mathrm{r}^{2}$ & CR & Y & Erro \\
\hline CE & 0,457 & 1,029 & $-0,11$ & 0,774 \\
MO & 0,771 & 1,081 & $-0,17$ & 0,041 \\
pH & 0,223 & 0,679 & 1,95 & 0,396 \\
\hline
\end{tabular}

Sendo: Var: variável, $\mathrm{r}^{2}$ : coeficiente de determinação, CR: coeficiente de regressão, Y: intercepto no eixo y, Erro: erro padrão previsto. 
Tabela 6. Erros médio absoluto dos métodos de interpolações krigagem, inverso da distância e normal da distância para os dados de $\mathrm{CE}\left(\mathrm{mS} \cdot \mathrm{m}^{-1}\right)$, $\mathrm{pH}$ e $\mathrm{MO}\left(\mathrm{g} \cdot \mathrm{kg}^{-1}\right)$ para as duas áreas experimentais. Table 6. Absolute mean errors of the kriging, inverse distance, and normal distance interpolation methods for the EC $\left(\mathrm{mS} . \mathrm{m}^{-1}\right), \mathrm{pH}$ and $\mathrm{MO}\left(\mathrm{g} \cdot \mathrm{kg}^{-1}\right)$ data for the two experimental areas.

\begin{tabular}{|c|c|c|c|c|c|c|}
\hline & \multicolumn{3}{|c|}{$\mathrm{T} 1$} & \multicolumn{3}{|c|}{ T2 } \\
\hline & $\mathrm{CE}$ & MO & $\mathrm{pH}$ & $\mathrm{CE}$ & MO & $\mathrm{pH}$ \\
\hline MK & 0,6873 & 0,0231 & 0,2810 & 0,5471 & 0,0277 & 0,3225 \\
\hline MI1 & 0,6697 & 0,0197 & 0,2826 & 0,5559 & 0,0243 & 0,2970 \\
\hline MI2 & 0,6679 & 0,0130 & 0,2846 & 0,5441 & 0,0176 & 0,3053 \\
\hline MI3 & 0,6928 & 0,0101 & 0,2911 & 0,5584 & 0,0145 & 0,3191 \\
\hline MI4 & 0,7144 & 0,0094 & 0,3009 & 0,5732 & 0,0136 & 0,3343 \\
\hline MI5 & 0,7280 & 0,0092 & 0,3112 & 0,5833 & 0,0134 & 0,3467 \\
\hline MN1 & 0,7343 & 0,0320 & 0,2881 & 0,6255 & 0,0363 & 0,2993 \\
\hline MN2 & 0,7502 & 0,0345 & 0,2918 & 0,6419 & 0,0388 & 0,3041 \\
\hline MN3 & 0,7587 & 0,0360 & 0,2952 & 0,6513 & 0,0402 & 0,3089 \\
\hline MN4 & 0,7642 & 0,0368 & 0,2983 & 0,6575 & 0,0411 & 0,3140 \\
\hline MN5 & 0,7680 & 0,0375 & 0,3012 & 0,6621 & 0,0418 & 0,3185 \\
\hline
\end{tabular}

Sendo: MK: erro absoluto médio da krigagem. MI1: erro absoluto médio do inverso da distância elevado a 1 a MI5: erro absoluto médio do inverso da distância elevado a 5. MN1: erro absoluto médio do normal da distância elevado a 1 a MN5: erro absoluto médio do normal da distância elevado a 5.

Analisando a Tabela 6 podemos observar que, de acordo com o erro absoluto médio, para a CE do talhão T1 e T2 o melhor método de interpolação foi o inverso da distância elevado a 2; para a MO do talhão T1 e T2 o melhor método de interpolação foi o inverso da distância elevado a 5 ; e para o pH do talhão $\mathrm{T} 1$ o melhor método de interpolação foi a krigagem, e para o talhão T2 o melhor método de interpolação foi o inverso da distância elevado a 1 (Tabela 6).

Observa-se que o maior erro absoluto médio da CE para o talhão T1 é de 0,77 , e para o talhão T2 é 0,67 , para a MO, o maior erro absoluto médio para o talhão $\mathrm{T} 1$ é de 0,38 , e para o talhão T2 é 0,42 , e para o $\mathrm{pH}$, o maior erro absoluto médio para o talhão T1 é de 0,32 , e para o talhão T2 é 0,35 . Os métodos de interpolações aplicados ao $\mathrm{pH}$ apresentaram erro médio inferior ao da $\mathrm{MO}$ e CE.

$\mathrm{Na}$ Tabela 7 é apresentada a raiz quadrada média do erro para as interpolações dos métodos de krigagem, inverso da distância e normal da distância, para as variáveis de condutividade elétrica, matéria orgânica e potencial hidrogeniônico para os talhões T1 e T2.

Analisando a Tabela 7, para a CE o melhor método de interpolação foi o inverso da distância elevado a 1 (talhão 1) e o inverso da distância elevado a 2 (talhão 2); para a MO foi o inverso da distância elevado a 5 (talhão 1 e 2); e para o pH foi a krigagem (talhão 1) e o inverso da distância elevado a 1 (talhão 2). Os métodos de interpolações aplicados a MO apresentaram valores de RMS inferiores ao pH e CE (Tabela 7).

Para a MO e o pH dos talhões T1 e T2, e para a CE do talhão T2, os menores erros encontrados, ou seja, o melhor modelo de interpolação foi semelhante quando analisado o MAE e o RMS, com exceção da condutividade do talhão T1, que para o MAE o melhor método foi o inverso distância elevado a 2, e para o RMS foi o inverso distância elevado a 1.

Aumentando a potência, tanto para o método inverso da distância e normal da distância, os erros tendem a serem maiores (Tabela 7) e os mapas gerados apresentam aspecto visual mais poluído. Conforme a Figura 13, podemos observar dois mapas do inverso da distância com potência diferente, o mapa da $5^{\circ}$ potência apresenta-se visualmente mais poluído que o da $1^{\mathrm{a}}$ potência, e com o aumento da potência observa-se que aumentam áreas com uma concentração maior na coloração.

Tabela 7. Raiz quadrada média do erro dos métodos de interpolações krigagem, inverso da distância e normal da distância para os dados de CE $\left(\mathrm{mS} . \mathrm{m}^{-1}\right)$, $\mathrm{pH}$ e $\mathrm{MO}\left(\mathrm{g} \cdot \mathrm{kg}^{-1}\right)$ para as duas áreas experimentais.

Table 7. Mean square root of the error of the interpolation methods kriging, inverse distance and normal distance for the data of EC $\left(\mathrm{mS} . \mathrm{m}^{-1}\right), \mathrm{pH}$ and $\mathrm{MO}\left(\mathrm{g} . \mathrm{kg}^{-1}\right)$ for the two experimental areas.

\begin{tabular}{rcccccc}
\hline & \multicolumn{3}{c}{$\mathrm{T} 1$} & & \multicolumn{3}{c}{$\mathrm{T} 2$} \\
& $\mathrm{CE}$ & $\mathrm{MO}$ & $\mathrm{pH}$ & $\mathrm{CE}$ & $\mathrm{MO}$ & $\mathrm{pH}$ \\
\hline RK & 0,9619 & 0,0324 & 0,3532 & 0,7638 & 0,0405 & 0,4094 \\
RI1 & 0,9387 & 0,0275 & 0,3547 & 0,7763 & 0,0358 & 0,3775 \\
RI2 & 0,9425 & 0,0182 & 0,3607 & 0,7577 & 0,0271 & 0,3887 \\
RI3 & 0,9829 & 0,0145 & 0,3738 & 0,7747 & 0,0238 & 0,4063 \\
RI4 & 1,0147 & 0,0137 & 0,3902 & 0,7939 & 0,0232 & 0,4251 \\
RI5 & 1,0342 & 0,0135 & 0,4054 & 0,8075 & 0,0232 & 0,4409 \\
RN1 & 1,0311 & 0,0454 & 0,3598 & 0,8698 & 0,0534 & 0,3788 \\
RN2 & 1,0545 & 0,0492 & 0,3648 & 0,8916 & 0,0573 & 0,3838 \\
RN3 & 1,0672 & 0,0514 & 0,3699 & 0,9040 & 0,0595 & 0,3892 \\
RN4 & 1,0752 & 0,0528 & 0,3745 & 0,9121 & 0,0611 & 0,3950 \\
RN5 & 1,0808 & 0,0538 & 0,3788 & 0,9182 & 0,0620 & 0,4003 \\
\hline S
\end{tabular}

Sendo: RK: raiz quadrada média do erro da krigagem. RI1: raiz quadrada média do erro do inverso da distância elevado a 1 a RI5: raiz quadrada média do erro do inverso da distância elevado a 5. RN1: raiz quadrada média do erro do normal da distância elevado a 1 a RN5: raiz quadrada média do erro do normal da distância elevado a 5.

\section{DISCUSSÃO}

A variação da CE no solo está relacionada ao teor de argila, umidade do solo, elementos químicos, presença de sais, teor de matéria orgânica, entre outros. Os valores máximo e mínimo de CE apresentaram uma grande amplitude, devido a variação de textura e teor de água, a MO e o $\mathrm{pH}$ apresentaram uma variação baixa entre os valores máximos e mínimos (Tabela 1).

Os valores de CE apresentaram variabilidade dos dados nos talhões, o que está relacionado com a variação da textura do solo, sendo que o talhão T1 apresenta textura mais heterogênea que o talhão T2. A grande amplitude entre os valores máximos e mínimos é também encontrada por Faulin (2005) em seu estudo sobre a variabilidade da condutividade elétrica do solo.

Os valores de MO tiveram pouca variação ao longo da área, o que representa que o manejo de sistema plantio direto é eficiente na manutenção da matéria orgânica no solo (Tabela 1). Favarato et al. (2015) encontrou valores semelhantes para a matéria orgânica do solo sob manejo de sistema plantio direto com palha de gramínea.

Os valores médios do $\mathrm{pH}$ do solo variaram pouco na área, o que indica que é aplicado um bom manejo do solo. Os valores de $\mathrm{pH}$ encontrados foram parecidos com os encontrados por Fontana et al. (2016) para Latossolos.

A CE apresentou maior coeficiente de variação em relação à $\mathrm{MO}$ e $\mathrm{pH}$, nas duas áreas, o que representa que os dados coletados apresentam uma maior variação. A MO e o pH apresentam coeficiente de variação baixo e a CE apresenta coeficiente de variação muito alto e alto, para os talhões T1 e T2, respectivamente, conforme a classificação de Gomes (1990).

O coeficiente de assimetria quantifica o desvio de uma distribuição, os valores observados demonstram distribuições 
assimétricas. Segundo Neves Neto et al. (2013) os valores de assimetria negativa demonstram existir tendência de concentração acima da média, sendo mais significativa quanto maior for o valor, em relação aos valores positivos a situação é inversa. A curtose caracteriza o achatamento da curva em relação à curva de distribuição, os dados analisados apresentam a curva com pico alto, ou seja, os dados observados estão próximos à média.

$\mathrm{O}$ modelo exponencial foi o que melhor se ajustou aos dados concorda com os resultados que o indicam como o de maior ocorrência para atributos do solo (NEVES NETO et al., 2013).

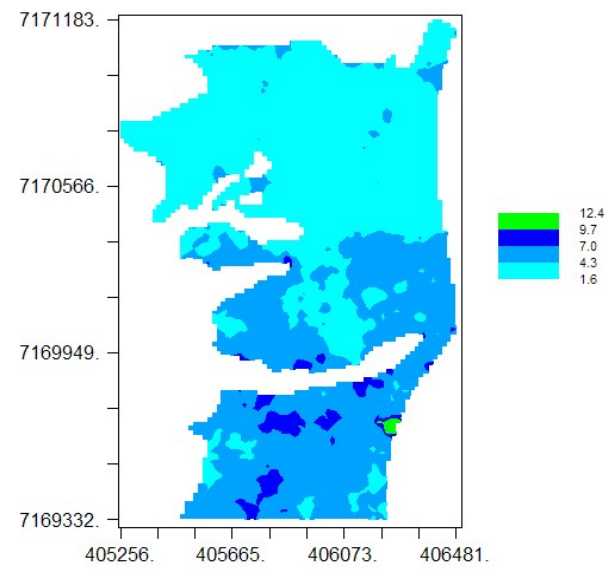

(a)

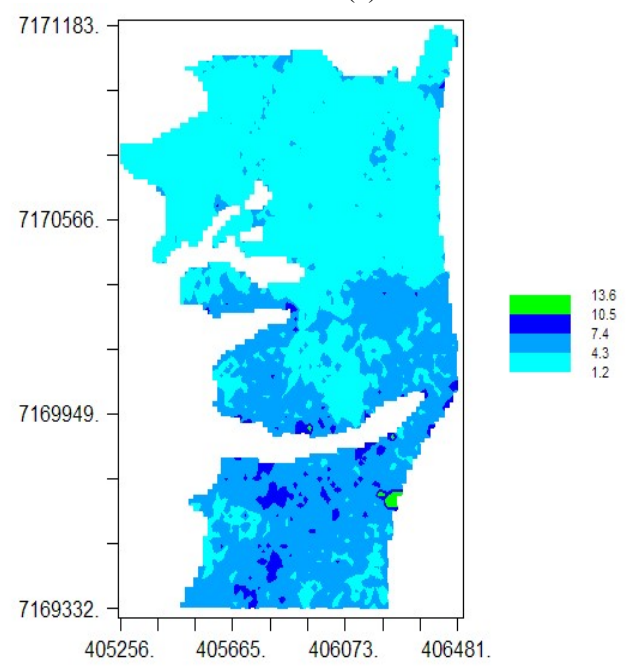

(b)

Figura 13. Mapa para a condutividade elétrica pelo inverso da distância elevado a 1 (a) e mapa para a condutividade elétrica pelo inverso da distância elevado a 5 (b).

Figure 13. Map for the electrical conductivity by the inverse of the elevated distance at 1 (a) and map for the electrical conductivity by the inverse of the distance elevated to 5 (b).

Segundo MATIAS et al. (2015) possuem distribuição aleatória os dados coletados a distâncias maiores que o alcance. Os valores de alcances dos dados analisados mostraram que a coleta foi realizada numa distância em que existe correlação entre os dados, sendo que as passadas poderiam ser em distâncias maiores que as que foram realizadas.

As distâncias de coletas de dados, em ambos os talhões, mostraram-se eficiente em relação ao alcance. De acordo com o estudo realizado por Souza et al. (2014) não é recomendado o uso de interpoladores, como a krigagem para calcular semivariogramas a partir de pontos muito espaçados, pois os mapas gerados não irão conduzir com a realidade da área. Em relação à Tabela 2, a $\mathrm{CE}, \mathrm{MO}$ e $\mathrm{pH}$ do solo do talhão $\mathrm{T} 1$ apresentam valores maiores de alcance que o talhão T2, demonstrando que o talhão T2 apresenta maior variabilidade dos atributos do solo que o talhão T1.

Todas as variáveis apresentaram efeito pepita baixo, o que indica que a amostragem foi eficiente. O grau de dependência espacial mostra para o talhão T1, o pH e a MO apresentam elevada dependência espacial, e a CE apresenta moderada dependência espacial, e para o talhão T2 o pH apresenta elevada dependência espacial, e a $\mathrm{CE}$ e a $\mathrm{MO}$ apresentam moderada dependência espacial, de acordo com a classificação de Lima et al. (2010).

$\mathrm{O}$ erro padrão previsto está relacionado ao erro padrão do coeficiente de regressão, a MO apresentou os menores erros, devido ao seu coeficiente de determinação ser maior. $\mathrm{O}$ pH apresentou erros menores que a $\mathrm{CE}$, mesmo tendo o coeficiente de determinação e o coeficiente de regressão inferiores.

Segundo Alves et al. (2012) o erro absoluto médio referese ao desvio médio absoluto dos valores interpolados em relação aos valores observados, e a raiz quadrada média do erro mede a variação dos valores estimados ao redor dos valores medidos; o ideal seria que os valores de MAE e RMS fossem igual a zero, ou o mais próximo de zero.

Analisando a Tabela 6 observamos que o MAE não apresenta uma amplitude com grande diferença entre os métodos de interpolações analisados, o que mesmo foi verificado por Yaguch et al. (2016) que em seu estudo, que o erro médio absoluto variou pouco no tempo e espaço.

Pinto et al. (2016) utiliza os erros MAE e RMS para analisar qual método gera um menor erro de previsão, e ele comprova que o MAE e o RMS são boas ferramentas para analisar o erro gerado pelo método e que eles auxiliam na escolha do melhor método de interpolação.

\section{CONCLUSÕES}

Os métodos de interpolação krigagem e inverso da distância se mostraram mais eficientes que o método normal da distância. Os menores erros de interpolação, para estimativa de $\mathrm{MO}, \mathrm{CE}$ e $\mathrm{pH}$ foram apresentados pelo método de interpolação inverso da distância para o talhão T2, no talhão T1 o método da Krigagem obteve os menores erros na determinação do $\mathrm{pH}$.

As distâncias de coleta dos dados mostraram-se adequadas, em relação ao alcance.

Quanto maior a potência de elevação, tanto para o método do inverso da distância quanto para normal da distância, os erros aumentam e também a poluição visual nos mapas.

Não é possível definir o modelo que apresenta menor erro de interpolação, pois o mesmo depende da variável que está sendo analisada.

\section{REFERENCIAS}

ALVES, E. D. L.; BIUDES, M. S.; VECCHIA, F. A. Z. Interpolação espacial na climatologia: análise dos critérios que precedem sua aplicação. Geonorte, Manaus, v. 3, n. 8, p. 606-618, 2012.

BERNARDI, A. C. de C.; BETTIOL, G. M.; GREGO, C. R.; ANDRADE, R. G.; RABELLO, L. M. E.; INAMASU R. Y. Ferramentas de agricultura de precisão como auxílio 
ao manejo da fertilidade do solo. Cadernos de Ciência e Tecnologia, Brasília, v. 32, n. 1-2, p. 205-221, 2015.

CARNEIRO, J. S. S.; SANTOS, A. C. M.; FIDELIS, R. R.; SILVA NETO, S. P.; SANTOS, A. C.; SILVA, R. R. Diagnóstico e manejo da variabilidade espacial da fertilidade do solo no cerrado do Piauí. Revista de Ciências Agroambientais, Alta Floresta, v. 14, n. 2, p. 10-21, 2016.

CHERUBIN, M. R.; SANTI, A. L.; EITELWEIN, M. T.; DA ROS; C. O.; BISOGNIN, M. B. Sampling grids used to characterize the spatial variability of $\mathrm{pH}, \mathrm{Ca}, \mathrm{Mg}$ and $\mathrm{V} \%$ in Oxisols. Revista Ciência Agronômica, Fortaleza, v. 45, p. 659-672, 2014. DOI: http://dx.doi.org/10.1590/S1806-66902014000400004

CHIG, L. A.; COUTO, E. G.; AMORIM, R. S. S. Tecnologias para levantamento da variabilidade dos atributos do solo para um programa de agricultura de precisão. Uniciências, Cuiabá, v. 14, n. 2. p. 127-149, 2015. DOI: http://dx.doi.org/10.17921/14155141.2010v14n2p\%25p

CORASSA, G. M.; AMADO, T. J. C.; TABALDI, F. M.; SCHWALBERT, R. A.; REIMCHE, G. B.; NORA, D. D.; ALBA, P. J.; HORBE, A. A. N. Espacialização em alta resolução de atributos da acidez de Latossolo por meio de sensoriamento em tempo real. Pesquisa Agropecuária Brasileira, Brasília, v. 51, n. 9, p. 13061316, 2016.

CUNHA, A. de M.; LANI, J. L.; DOS SANTOS, G. R.; FERNANDES FILHO, E. I.; TRINDADE F. S.; DE SOUZA, E. Espacialização da precipitação pluvial por meio de krigagem e cokrigagem. Pesquisa Agropecuária Brasileira, Brasília, v. 48, n. 9, p. 1179-1191, 2013.

EMBRAPA_Empresa Brasileira de Pesquisa Agropecuária Centro Nacional de Pesquisa de Solos. Sistema brasileiro de classificação de solos. Rio de Janeiro: Embrapa, 2012. p. 412.

FAULIN, G. D. C. Variabilidade espacial do teor de água e sua influência na condutividade elétrica do solo. 2005. 53f. Dissertação (Mestrado em Agronomia) Escola superior de agricultura Luiz de Queiroz, Universidade de São Paulo, Piracicaba, 2005.

FAVARATO, L. F.; DE SOUZA, J. L.; GALVÃO, J. C. C.; DE SOUZA, C. M.; GUARÇONI, R. C. Atributos químicos do solo sobre diferentes plantas de cobertura no sistema plantio direto orgânico. Revista Brasileira de Agropecuária Sustentável, Viçosa, v. 5, n. 2, p. 19-28, 2015. DOI: https://doi.org/10.21206/rbas.v5i2.312

FONTANA A.; TEIXEIRA, W. G.; BALIEIRO, F. D. C.; MOURA, T. P. A. D.; MENEZES, A. R. D.; SANTANA, C. I. Characteristics and attributes of Oxisols under different land uses in the western region of the state of Bahia, Brazil. Pesquisa Agropecuária Brasileira, Brasília, v. 51, n. 9, p. 1457-1465, 2016. DOI: http://dx.doi.org/10.1590/s0100-204x2016000900044

GOMES, F. P. Curso de estatística experimental. 12. ed. Piracicaba: Nobel, 1990. 467 p.

KODAIRA, M.; SHIBUSAWA, S. Using a mobile real-time soil visible-near infrared sensor for high resolution soil property mapping. Geoderma, Amsterdam, v. 199, p. 6479 , 2013.

DOI: https://doi.org/10.1016/j.geoderma.2012.09.007

LEGATES, D. R.; MCCABE JR, G. J. Evaluating the use of "goodness-of-fit" measures in hydrologic and hydroclimatic model validation. Water resources research, Washington, v. 35, n. 1, p. 233-241, 1999. DOI: https://doi.org/10.1029/1998WR900018

LIMA, J. S. de S.; SOUZA, G. S. de; SILVA, S. A. Amostragem e variabilidade espacial de atributos químicos do solo em área de vegetação natural em regeneração. Revista Árvore, Viçosa, v. 34, n. 1, p. 127 136, 2010. DOI: http://dx.doi.org/10.1590/S010067622010000100014

MACHADO, P. L. O. D. A.; BERNARDI, A. C. D. C.; VALENCIA, L. I. O.; MOLIN, J. P.; GIMENEZ, L. M.; SILVA, C. A.; MEIRELLES, M. S. P. Electrical conductivity mapping in relation to clay of a Ferralsol under no tillage system. Pesquisa Agropecuária Brasileira, Brasília, v. 41, n. 6, p. 1023-1031, 2016. DOI: http://dx.doi.org/10.1590/S0100-204X2006000600019

MANZATTO, C. V.; BHERING, S. B.; SIMÕES, M. Agricultura de precisão: propostas e ações da Embrapa Solo. Embrapa Solos, 1999. Disponível em: <http://www.cnps.embrapa.br>. Acesso em: 15 jun. 2006.

MATIAS, S. S. R.; NÓBREGA, J. C. A; NÓBREGA, R. S. A.; ANDRADE, F. R.; BAPTISTEL, A. C. Variabilidade espacial de atributos químicos em Latossolo cultivado de modo convencional com soja no cerrado piauiense. Revista Agroambiente On-line, Boa Vista, v. 9, n. 1, p. 17-26, 2015.

MOLIN, J. P.; AMARAL, L. R.; COLAÇO, A. F. Agricultura de precisão. 1. ed. São Paulo: Oficina de textos. 240 p.

NEVES NETO, D. N.; SANTOS, A. C.; DOS SANTOS, P. M.; MELO, J. C.; SANTOS, J. S. Análise espacial de atributos do solo e cobertura vegetal em diferentes condições de pastagem. Revista Brasileira de Engenharia Agrícola e Ambiental, Campina Grande, v. 17, n. 9, p. 995-1004, 2013. DOI: http://dx.doi.org/10.1590/S1415-43662013000900013

PINTO, J. M.; LOBO, V. G.; GREER, V. R. A. Operadores do comércio: um estudo da aplicação de modelos univariados à previsão de admissões. Revista UNIABEU Belford Roxo, Rio de Janeiro, v. 9, n. 21, p. 72-87, 2016.

SOUZA, Z. M. D.; SOUZA, G. S. D.; MARQUES JR, J.; PEREIRA, G. T. Número de amostras na análise geoestatística e na krigagem de mapas de atributos do solo. Revista Ciência Rural, Santa Maria, v. 44, n. 2, p. 261-268, 2014. DOI: http://dx.doi.org/10.1590/S010384782014000200011

WILLMOT, C. J.; ACKLESON, S. G.; DAVIS, J. J.; FEDDEMA, K. M.; KLINK, K. M.; LEGATES, D. R.; O'DONNELL, J.; ROWE, C. M. Statistics for the evaluation and comparison of models. Journal of Geophysical Research, Washington, v. 90, n. C5, p. 8995-9005, 1985.

DOI: https://doi.org/10.1029/JC090iC05p08995

YAGUCHI, S. M.; MASSIGNAM, A. M.; DA SILVA, R. W.; PANDOLFO, C. Preenchimento de Falhas dos Dados Diários de Temperatura Máxima e Mínima do Ar. Revista Ciência e Natura, Santa Maria, v. 38, n. 3, p. 1419-1425, 2016.2 DOI: http://dx.doi.org/10.5902/2179460X19502 